\title{
AU SUJET LE L'ARTIGLE DE M. K. KATRANDJIEFF SUR LA PASTEURISATION A BASSE TEMPÉRATURE
}

\author{
par Chr. BARTHEL
}

Dans son travail : Nouvelles recherches sur la pasteurisation à basse température (Le Lait, 1930, p. 129) M. KatRAndJIEFF dit que « BARTHEL et STenström d'une part, et VAN DE SLuIs, d'autre part, croient que pour détruire la virulence du lait tuberculeux, une température de $80^{\circ} \mathrm{C}$., agissant pendant une heure, est indispensable $n$.

Dans le travail cité de Barthel et Stenström (Centralbl. für Bakt., I. Abt., 30, 1901, 429) nous n'avons rien dit au sujet d'un chauffage pendant une heure à $80^{\circ} \mathrm{C}$. Il s'agissait d'un lait tuberculeux très floconneux, qui coagulait complètement à $80^{\circ} \mathrm{C}$. et dans lequel les bacilles tuberculeux se montraient virulents même après un chauffage de 10 min. à $80^{\circ} \mathrm{C}$. Plus tard, nous avons démontré, ainsi que l'ont fait BANG, THeoBALD Sмrтн et d'autres, la grande influence de l'état physique du lait dans ce genre d'expériences (voir Centralbl. für Bakt., I. Abt., 6, 1904, 459)

Au sujet dela pasteurisation lente, ou basse, Barthel et STEnström ont démontré en 1917 (Zeitschr. f. Gärungsphysiologie, 6, 110) que le chauffage à $63^{\circ} \mathrm{C}$. pendant 20 à $30 \mathrm{~min}$, dans les conditions pratiques était pleinement suffisant pour rendre inoffensif le lait tuberculeux.

\section{BIBLIOGRAPHIE ANALYTIQUE}

\section{LES LIVRES}

\section{Liste of publications of the Bureau of Dairy Industry from} July 1, 1924 to Dec. 31, 1928. - United States department of agriculture. Bureau of dairy industry. Une brochure dactylographiée de 26 pages.

Cette publication s'ajoute à celle que si heureusement le Ministère de l'Agriculture des Etats-Unis fait parâtre de temps à autro. Il s'agit cette fois des publications du Bureau de l'industrie laitière, bureau maintenant indépendant, séparé de calui de l'industrie animale, et qui ont vu le jour aux Etats-Unis du $1^{\text {er }}$ juillet 1924 au 31 décembre 1928.

Au point de vue bibliographique, de telles publications sont de la plus haute importance, et e'est faciliter le travail de recherches que d'avoir en main un pareil document.

Ch. P.

\section{P. Cottin. - La Cochinchine. Etude zooéconomique (Notes d'épi-} démiologie). Thèse Doct. Vét., Paris, 1927. Brochure de 84 pages.

Les vétérinaires qui vivent dans les colonies ne manquent pas de joindre à laurs études zootechniques des considérations sur l'épidémiologie. On peut en deviner le grand intérêt, car les maladies pèsent très fortement sur l'élevage et il importe d'abord de les combattre si l'on veut améliorer celui-ci.

Ch. P. 